\title{
O ASPECTO CONSERVADOR DA FORMAÇÃO EM CONTABILIDADE NO AMBIENTE DE AVERSÃO À PERDA DIANTE DE VALORES CULTURAIS
}

\section{CONSERVATIVE ASPECT OF ACCOUNTING DEGREE IN LOSS AVERSION ENVIRONMENT IN THE FACE OF CULTURAL VALUES}

\section{ASPECTO CONSERVADOR DE LA FORMACIÓN EN CONTABILIDAD EN EL AMBIENTE DE AVERSIÓN A LA PÉRDIDA ANTE DE LOS VALORES CULTURALES}

Recebido em: 13-07-2020

Avaliado em: 20-01-2021

Reformulado em: 01-02-2021

Aceito para publicação em: 28-07-2021

Publicado em: 26-11-2021

Editor Responsável: Franciele Beck

\author{
Anderson José Freitas de Cerqueira ${ }^{1}$ \\ César Valentim de Oliveira Carvalho Júnior ${ }^{2}$ \\ José Maria Dias Filho ${ }^{3}$
}

\section{RESUMO}

A literatura indica que as perdas possuem uma aparência emocional maior do que os ganhos, além de apontar que as pessoas de diferentes orientações culturais podem tomar decisões distintas. Este artigo tem como objetivo investigar se as dimensões culturais impactam as decisões dos profissionais da área gerencial, que possuem graduação em Ciências Contábeis, em um ambiente de aversão à perda. A coleta de dados ocorreu por meio da aplicação de questionários para estudantes que estão cursando pósgraduação lato sensu em áreas ligadas à gestão. O tratamento estatístico se deu por meio de regressão linear múltipla pelo Método dos Mínimos Quadrados Ordinários e teste não paramétrico de MannWhitney. Os resultados indicaram que as dimensões culturais Aversão à Incerteza e Masculina/Feminina, relacionadas com práticas conservadoras, impactam, significativamente, no grau de aversão à perda dos profissionais da área gerencial que possuem formação em contabilidade, em relação aos que possuem formação em outras áreas. Adicionalmente, os resultados indicaram que o nível de aversão à perda é maior para os indivíduos do sexo masculino, independentemente do aspecto cultural. Observa-se que a raiz biológica no comportamento conservador, juntamente com o ambiente cultural, resgatam o viés emocional dos profissionais com graduação em Ciências Contábeis, que podem ser influenciados pelos princípios e pelas normas e práticas. Esta pesquisa possui um viés inovador em considerar o aspecto conservador dos profissionais da área gerencial, graduados em Ciências Contábeis, diante de valores culturais. Todavia, como pesquisa exploratória, o estudo possui limitação de tamanho da amostra e amplitude geográfica. Essas observações, em tempo, servem como sugestão para estudos subsequentes.

\footnotetext{
${ }^{1}$ Doutorando em Administração pela UFBA; Bolsista de Produtividade do Centro Universitário Estácio da Bahia; E-mail: andersonjose.freitas@gmail.com

${ }^{2}$ Doutor em Contabilidade pela FEA/USP; Professor Adjunto da Faculdade de Ciências Contábeis da UFBA; E-mail: cesarvalentim@ufba.br

${ }^{3}$ Doutor em Contabilidade pela FEA/USP; Professor Adjunto da Faculdade de Ciências Contábeis da UFBA; E-mail: zemariadias@uol.com.br
} 
Palavras-chave: Aversão à Perda. Conservadorismo. Valores Culturais. Contabilidade.

\section{ABSTRACT}

The literature indicates that losses have a greater emotional outlook than gains, and points out that people from guidelines different cultures can make different decisions. This article aims to investigate if the cultural dimensions impact the decisions of professionals among managers who graduated in accounting in an environment of loss aversion. Data collection was carried out through the application of questionnaires to students who are studying lato sensu post-graduation in areas related to management. The statistical treatment was given by multiple linear regression by the Ordinary Least Squares method and Mann-Whitney non-parametric test. The results indicated that the cultural dimensions Aversion to Uncertainty and Masculine / Feminine, related to conservative practices, significantly impact on the degree of loss aversion of professionals among managers who graduated in accounting. Additionally, the results indicated that the level of loss aversion is greater for males, regardless of the cultural aspect. It is observed that the biological root in conservative behavior, and the cultural environment, rescues the emotional bias of professionals who graduated in accounting, who are influenced by principles and norms, and practices. This study has an innovative bias for considering the conservative aspect of professionals among managers who graduated in accounting before cultural values. However, as exploratory research, it has a limited sample size and geographical amplitude. These observations, in time, serve as a suggestion for subsequent studies.

Keywords: Loss Aversion. Conservatism. Cultural Values. Accounting.

\section{RESUMEN}

La literatura indica que las pérdidas tienen una apariencia emocional mayor que las ganancias, además de apuntar que las personas de diferentes culturas pueden tomar decisiones distintas. Este artículo tiene como objetivo investigar si las dimensiones impactan las decisiones de los profesionales de la gestión formados en contabilidad en un ambiente de aversión a la pérdida. La recolección de datos ocurrió a través de la aplicación de cuestionarios para estudiantes del postgrado lato sensu en áreas vinculadas a contabilidad de la gestión. El tratamiento estadístico se dio vía regresión lineal múltiple por el Método de los Mínimos Cuadrados Ordinarios y prueba no paramétrica de Mann-Whitney. Los resultados indicaron que las dimensiones culturales Aversión a la Incertidumbre y Masculina / Femenina, relacionadas con las prácticas conservadoras, impactan, significativamente, en el grado de aversión a la pérdida de los profesionales de la gestión formados en contabilidad. Además, los resultados indicaron que el nivel de aversión a la pérdida es mayor para los individuos del género masculino, independientemente del aspecto cultural. Se observa que la raíz biológica en el comportamiento conservador, junto con el ambiente cultural, rescata el sesgo emocional de los profesionales formados en contabilidad, que son influenciados por los principios y las normas y prácticas. Este estudio tiene un sesgo innovador por la premisa de considerar el aspecto conservador de los profesionales de la gestión formados en contabilidad antes de que los valores culturales. Sin embargo, como investigación exploratoria, tiene limitación de tamaño de la muestra y amplitud geográfica. Estas observaciones, a tiempo, sirven como sugerencia para estudios posteriores.

Palabras clave: Aversión a la Pérdida. Conservadorismo. Valores Culturales. Contabilidad. 


\section{INTRODUÇÃO}

Estudos sobre o comportamento humano vêm crescendo nas últimas décadas, sobretudo os vieses emocionais, psicológicos e cognitivos que impactam diretamente as decisões sob a incerteza dos indivíduos, em ambientes com riscos nos ganhos e, principalmente, nas perdas. Para Rouziès, Segalla e Besson (1999), a perda é uma das áreas mais estudadas dentro da psicologia cognitiva e economia comportamental.

Dickhaut, Basu, McCabe e Waymire (2010), que apresentam o conservadorismo como um padrão humano e o cérebro como a instituição contábil original, destacam que a aversão à perda pode ser prevista pelo nível de ativação cerebral no córtex pré-frontal. Os autores sugerem que as explicações para os princípios contábeis ligados ao conservadorismo podem derivar de como os ganhos e as perdas são processadas, diferencialmente, pela atividade neural. Kahneman e Tversky $(1979,1983)$ e Tversky e Kahneman (1991) salientam que um dos fenômenos básicos da escolha sob risco e incerteza é que as perdas possuem um aspecto atrativo maior do que os ganhos.

Sob esse ambiente, Kahneman e Tversky (1979) e Tversky e Kahneman (1992) ressaltam que o problema da interpretação do risco é observado de duas formas: primeiramente, os indivíduos preferem uma pequena probabilidade de ganhar um grande prêmio sobre o valor esperado: em segundo, há uma propensão ao risco prevalente quando os indivíduos devem escolher entre uma perda amarga (pequena) e uma probabilidade de uma perda maior.

Assim, para Novemsky e Kahneman (2005b) e Sokol-Hessner, Hsu, Curley, Delgado, Camerer e Phelps (2009), quando há risco, a emoção desempenha um papel crucial na aversão à perda. Contudo, um aspecto que impacta o comportamento emocional do indivíduo - embora pouco explorado na literatura sobre aversão à perda, principalmente brasileira, - é a cultura. Para Wang, Rieger e Hens (2016), a emoção tem grande impacto na aversão à perda e, portanto, é concebível que as pessoas de diferentes culturas tenham decisões diferentes sob esta condição, por causa dos diferentes hábitos que regulam as emoções. Isso se deve ao fato de os indivíduos expressarem as emoções de acordo com suas as orientações sociais e culturais (Le Breton, 2009; Miguel, 2015).

Os países também podem ser diferenciados por dimensões culturais (Hofstede, 1991; Hofstede, Hilal, Malvezzi, Tanure, \& Vinken, 2010). Por isso, diversos pesquisadores têm mostrado como estas dimensões culturais, apesar de não representarem a cultura de um povo em sua totalidade (Joannidàs, Wickramasinghe, \& Berland, 2012), identificam suas características.

Especificamente, tem sido notado que nas últimas décadas, os pesquisadores de contabilidade geralmente tentaram integrar as diferenças entre culturas na explicação do comportamento gerencial e em vários fenômenos contábeis (Khlif, 2016).

Sob o ponto de vista da relação entre o conservadorismo, risco, cultura e formação em Contabilidade há poucos estudos. Chand, Cummings e Patel (2012) identificaram que a cultura nacional influencia a interpretação dos alunos e a aplicação de expressões de incerteza relacionadas ao reconhecimento de ativos e passivos e suas divulgações. Os resultados mostram que os estudantes chineses exibem maior conservadorismo em relação aos estudantes australianos.

Com profissionais de Contabilidade, Schultz e Lopez (2001) investigaram os julgamentos feitos pelos contadores (as) ao determinar estimativas contábeis referentes às despesas de garantia na França, Alemanha e Estados Unidos. Os resultados indicaram que contadores (as) americanos exibem menos aversão ao risco, já que as despesas de garantia são mais baixas em comparação aos contadores (as) franceses (as) e alemães (as). Já Patel, Harrison e McKinnon (2002) encontraram influências culturais em julgamentos profissionais da área contábil que atuam na Austrália, Índia, China e Malásia referente a conflitos entre auditor e cliente. 
Soeters e Schreuder (1988) e Chow e Hwang (1995) utilizaram as dimensões culturais de Hofstede para identificar que existe efeito cultural dos Estados Unidos sobre empresas contábeis da Holanda e Taiwan, respectivamente. Busco e Scapens (2011) identificaram a influência do contexto cultural nos sistemas de contabilidade gerencial. Ahrens e Mollona (2007), inclusive, propuseram a cultura como uma prática a ser abordada na área gerencial.

Doupnik e Richter (2004) evidenciaram que, na maioria dos casos, os contadores alemães parecem ser mais conservadores em relação aos americanos, pois exibem um viés conservador na interpretação da palavra "provável", usada nas normas internacionais de Contabilidade.

No mesmo sentido, Doupnik e Riccio (2006) conduziram um estudo, sob o âmbito cultural comparativo entre os Estados Unidos da América (EUA) e o Brasil. Os autores notaram que os contadores (as) brasileiros (as) parecem adotar uma abordagem mais conservadora ao reconhecer elementos que aumentam o resultado em comparação com os contadores (as) dos EUA.

Entretanto, este resultado, talvez, não represente a totalidade dos profissionais formados em Contabilidade, em razão do Brasil ser um país com características culturais peculiares em suas regiões. Trata-se do quinto maior país do mundo, tanto em termos de superfície quanto de tamanho da população. Ocupa metade da América do Sul e é altamente diversificado dentro de suas fronteiras em termos de condições climáticas, vegetação, etnias, linguagens, comidas, música, ritos sociais e desenvolvimento econômico (Hofstede et al., 2010).

Sendo assim, se a cultura $(p)$ pode influenciar a emoção $(q)$, e a última pode exercer influência no nível de aversão à perda $(r)$, então há um silogismo hipotético válido, em que se $(p \rightarrow q)^{\wedge}(q \rightarrow r)$, então $p \rightarrow r$. Diante deste argumento lógico, cabe ressaltar que o ser humano (com formação ou não em Contabilidade), biologicamente, é conservador. Todavia, surge a necessidade de compreender se o indivíduo com formação em Contabilidade, diante desta característica biológica que transcende às práticas contábeis (Dickhaut, 2009; Dickhaut et al., 2010), mantém ou não o viés do conservadorismo diante de seus valores culturais.

A justificativa do estudo refere-se à identificação de uma lacuna nas discussões sobre o aspecto cultural como um elemento capaz de influenciar as emoções dos indivíduos, que, neste espectro, pode refletir nas decisões gerenciais daqueles que possuem formação em Contabilidade, que podem manter o habitus do ser conservador. Isto porque, poucos estudos abordam a dimensão cultural na Contabilidade Gerencial e no controle organizacional (Ahrnes \& Mollona, 2007).

Ressalta-se que neste estudo, a captura do nível de conservadorismo é através do nível de aversão à perda, pois uma implicação da aversão à perda é que os indivíduos têm uma forte tendência a permanecer no status quo (conservador), porque as desvantagens de deixá-lo parecem maiores do que as vantagens (Kahneman, Knetsch, \& Thaler, 1991).

Até a presente data, não há nenhuma pesquisa que considere o profissional da área gerencial que possui formação em Contabilidade, indicando um comportamento com viés conservador em um ambiente de aversão à perda, que pode sofrer influência do ambiente cultural.

Ressalta-se que há muitos estudos que investigaram a relação entre o nível de aversão à perda nas decisões de riscos de indivíduos que possuem contato com a área. No contexto nacional, Melo e Silva (2010) destacam-se como um dos primeiros trabalhos que investigaram o impacto do viés da aversão à perda entre estudantes e profissionais de Contabilidade. Os autores notaram que há indícios que a ocupação promove maior grau de aversão à perda.

Portanto, torna-se necessário compreender e avançar nesse tema, tendo em vista que os profissionais que utilizam meios da Contabilidade Gerencial (orçamento, medida de desempenho, gestão de custos, etc.) são suscetíveis a serem influenciados por questões emocionais, enquanto os formados em 
contabilidade podem ter uma tendência adicional conservadora nas decisões, que podem ser provocados pelo contexto cultural. Como consequência, sistemas de contabilidade gerencial e práticas de gestão que são eficazes em um ambiente podem ser ineficazes em outros ambientes (Tsui, 2001).

Neste estudo, destaca-se que os profissionais da Contabilidade (Gerencial e Financeira) possuem a mesma formação acadêmica, baseada nos princípios contábeis, em que a prudência exerce um papel de destaque, visto que norteia a ideia de apenas aceitar o reconhecimento antecipado de perdas, exigindo maior materialidade dos ganhos.

Apesar dos profissionais da Contabilidade Gerencial, na sua atuação, incorporarem diversos conhecimentos e tecnologias da área de gestão, a formação contábil básica pode funcionar como um reforço à aversão à perda inata. Ademais, tal constatação reforçaria a necessidade de incorporar a discussão da economia comportamental na formação em Contabilidade Gerencial, destacando os perigos dos vieses cognitivos e emocionais no processo decisório.

Considerando que os valores culturais podem influenciar as emoções, bem como interferir no perfil biológico conservador em decisões de risco dos profissionais formados em Contabilidade, tem-se a seguinte questão de pesquisa: Até que ponto os valores culturais dos profissionais que atuam na área gerencial influenciam as decisões em um ambiente de aversão à perda?

$\mathrm{O}$ artigo tem como base o estudo de Wang et al. (2016): "The Impact of Culture on Loss Aversion", sendo, porém, aplicado especificamente em estudantes de pós-graduação lato sensu de cursos relacionados à área contábil, que atuam na área gerencial profissionalmente. O objetivo desta pesquisa é investigar se o profissional formado em Contabilidade mantém ou potencializa a característica biológica conservadora diante de valores culturais em um ambiente de aversão à perda. Além disso, contribuir para literatura, sobretudo nacional, sobre o contexto cultural ser um vetor importante a ser considerado para o profissional da área de Contabilidade Gerencial nas decisões que envolvam risco, ponderando a característica conservadora da formação acadêmica.

Consideramos que isto vem reforçar a necessidade de novas investigações em torno do tema, haja vista as reconhecidas diferenças culturais existentes no Brasil, sobretudo no que diz respeito às crenças, comportamentos, valores, costumes, religião e folclore. Em que pesem as tentativas de se criar uma sociedade homogênea, principalmente por meio da globalização e dos meios de comunicação de massa, entendemos que as diferenças culturais resistem e seguem exercendo influência na percepção dos indivíduos, na forma de pensar, de agir e reagir.

Ao lado disso, há de se considerar que os contadores vêm sendo cada vez mais pressionados a participar do processo decisório, avaliando riscos, identificando alternativas de decisão e ajudando a construir os resultados no atual ambiente de negócios. Assim, compreender como eles se relacionam com os riscos e se comportam ante as possibilidades de perda parece-nos fundamental para se ajustar o próprio processo de formação. Do contrário, pode-se estabelecer um hiato ainda mais significativo entre as competências e habilidades do contador e as demandas a que esse profissional será submetido em seu cotidiano profissional.

O trabalho expõe cinco partes, além desta introdução. A próxima seção trata de um breve referencial teórico que alinha a construção das hipóteses. A terceira seção apresenta a metodologia utilizada. A quarta seção expõe as análises dos resultados, seguida da conclusão e recomendações.

\section{FUNDAMENTAÇÃO TEÓRICA E HIPÓTESES}


Nesta seção abordam-se as principais considerações acerca dos aspectos culturais, aprofundando em cada dimensão elaborada por Hofstede (1990). Em seguida, apresenta-se fundamento relacionado à emoção e aversão à perda que são impactadas pela cultura com viés conservador. Por fim, a referência teórica que aborda o aspecto conservador dos profissionais de contabilidade. Todos esses tópicos direcionam à construção das hipóteses.

\subsection{Dimensões Culturais de Hofstede}

Cultura, na visão de Schein (1997), é o padrão de premissas básicas que o grupo desenvolveu visando à solução de seus problemas de adaptação externa e de integração interna, que tem funcionado suficientemente bem para ser considerado válido e, por essa razão, pode ser visto pelos novos membros como a maneira correta de perceber, pensar e sentir em relação a estes problemas. Diante da diversidade de aspectos no ambiente externo e interno que impactam a cultura dos grupos, pesquisadores a consideram nos estudos entre países. Hofstede et al. (2010, p.336), por sua vez, descreveram a cultura como "a programação coletiva dos espíritos que distingue os membros de um grupo humano do outro" e definiram quatro dimensões culturais, às quais foi posteriormente adicionada à uma nova dimensão, resultando em cinco dimensões culturais, conforme a Tabela 1.

\section{Tabela 1}

Dimensões culturais de Hofstede

\begin{tabular}{|c|c|c|}
\hline Dimensões Culturais & Sigla & Significado \\
\hline Distância do Poder & PDI & $\begin{array}{l}\text { Trata-se do nível de reação contra desigualdade social, ou seja, como a } \\
\text { sociedade reage em relação à distância entre as classes sociais. Quanto maior o } \\
\text { PDI, maior o nível de aceitação à desigualdade social. }\end{array}$ \\
\hline Aversão à Incerteza & UAI & $\begin{array}{l}\text { Refere-se ao nível de incerteza que a sociedade possui sobre o futuro. Nessa } \\
\text { dimensão, a sociedade pode preferir tomar iniciativas a fim de controlar o } \\
\text { futuro ou deixar acontecer naturalmente. Quanto maior o UAI, maior a aversão } \\
\text { à incerteza. }\end{array}$ \\
\hline Individualismo / Coletivismo & IDV & $\begin{array}{l}\text { Mede o nível de relações interpessoais que o indivíduo possui. A sociedade } \\
\text { mais individualista tem um foco maior nas tarefas a serem realizadas e menor } \\
\text { nas relações interpessoais. Já no coletivismo, os grupos favorecem o trabalho } \\
\text { em equipe e tendem a atingir os objetivos do grupo ao invés dos objetivos } \\
\text { individuais. Quanto maior o IDV, maior o individualismo. }\end{array}$ \\
\hline Masculinidade / Feminilidade & MAS & $\begin{array}{l}\text { Dimensiona a divisão dos papéis emocionais e sociais entre homens e mulheres. } \\
\text { Quanto mais masculina, mais competitiva, enquanto quanto mais feminina, } \\
\text { mais cooperativa. Quanto maior o MAS, mais masculina é a sociedade. }\end{array}$ \\
\hline $\begin{array}{l}\text { Orientação de Curto / Longo } \\
\text { Prazo }\end{array}$ & LTO & $\begin{array}{l}\text { Quantifica o grau de preocupação de uma sociedade com o futuro em oposição } \\
\text { à obtenção de resultados rápidos. Quanto maior o LTO, mais orientada a planos } \\
\text { de longo prazo é a sociedade. }\end{array}$ \\
\hline
\end{tabular}

Nota. Adaptado de Hofstede et al. (2010).

Diversos estudiosos utilizam os questionários de Hofstede para compreender as dimensões culturais em países. Embora, para Hofstede et al. (2010), as dimensões culturais não se aplicam somente à divisão de países, mas também devem se aplicar a diferentes regiões de um país. Para estes autores, ao desenvolver um estudo sobre as dimensões culturais entre regiões no Brasil, a cultura brasileira é o 
resultado da integração e absorção de civilizações distintas dentro de um núcleo cultural de língua portuguesa. Os autores identificaram, através das dimensões de Hofstede et al. (2010), conforme a Tabela 2, que no Brasil há diferenças culturais significativas entre as regiões em relação à média do país.

Tabela 2

Dimensões culturais nas regiões brasileiras

\begin{tabular}{lccccc}
\hline \multicolumn{1}{c}{ Região/País (número de estados) } & PDI & UAI & IDV & MAS & LTO \\
Sul (3) & 4 & -3 & 6 & 3 & 2 \\
Sudeste (5) & 2 & 1 & 2 & -5 & -3 \\
Centro-oeste (3) & -1 & -4 & 0 & 0 & 1 \\
Nordeste (8) & -3 & 5 & -1 & -3 & 0 \\
Norte (4) & 0 & -7 & -5 & 10 & 2 \\
\hline \hline Média Brasil & 69 & 76 & 38 & 49 & 44 \\
\hline
\end{tabular}

Nota. PDI = Distância do Poder; UAI = Aversão à Incerteza; IDV = Individualismo / Coletivismo; MAS = Masculinidade / Feminilidade; LTO = Orientação de Curto / Longo Prazo. Adaptado de Hofstede et al. (2010). Os pontos apresentados pelos estados referem-se ao aumento ou diminuição em relação à média do Brasil.

Vale ressaltar que este artigo se refere à dimensão cultural da cidade de Salvador, capital da Bahia, que representa a proposta de investigação deste estudo. Este estado, conforme salientado por Hofstede et al. (2010), apresentou um resultado semelhante ao da região sul. Especificamente em Salvador, Rebequi (2015) identificou similaridades com as dimensões culturais do sul e sudeste.

Considerar aspectos culturais torna-se relevante diante da influência sobre os profissionais que atuam na área gerencial. No processo de sistema de controle de gestão, a cultura tem dois efeitos importantes para Birnberg e Snodgrass (1988). Pode afetar a escolha dos estímulos aos quais o indivíduo segue, ou pode afetar quaisquer julgamentos de valor sobre os estímulos.

Para Gray (1988), a maioria dos estudos de contabilidade relacionados à cultura tem usado o modelo dimensional de Hofstede para identificar fatores culturais que são suscetíveis de ser associados com as práticas contábeis.

Segundo Hofstede (1991), a cultura é uma programação mental em que cada pessoa, quando nasce, recebe em sua carga genética as características da natureza humana, mas durante a sua vida será programada mentalmente de acordo com o meio em que vive. Diante disso, é possível conjecturar que a cultura local pode influenciar com o aspecto emocional dos indivíduos, tendo como viabilidade as proxies desenvolvidas por Hofstede.

\subsection{Cultura, Emoção e Aversão à Perda}

Inicialmente, no modelo econômico clássico, o indivíduo era visto como um homem econômico, em que as decisões eram conduzidas somente pela racionalidade. Contudo, Para Tvsersky e Kahneman (1991), esse modelo é irrealista, uma vez que os indivíduos fazem as suas escolhas em um contexto abrangente, incorporando todos os detalhes relevantes da situação atual, bem como as expectativas futuras sobre todas as oportunidades e riscos. Esse pensamento pode ser complementado pela investigação de Li, Kenrick, Griskevicius e Neuberg (2012) que, baseada na psicologia e economia experimental, demonstra que os fatores psicológicos muitas vezes levam à aparente irracionalidade e limitações nos processos de julgamento e tomada de decisão. 
Já Thaler (2001) direciona que aversão à perda é uma anomalia da aversão ao risco não prevista pelo modelo clássico do agente econômico. O autor expõe que, emocionalmente, o sentimento da dor de uma perda é mais agudo do que o prazer de um ganho equivalente, principalmente quando a contabilidade mental dos indivíduos processa ações individuais (Barberis \& Huang, 2001).

Em outro estudo Thaler e Sunstein (2008) identificaram que a aversão à perda funciona como uma espécie de empurrão cognitivo, pressionando o indivíduo a não fazer alterações nas decisões, mesmo que essas mudanças sejam do interesse do indivíduo. Esta dificuldade existe porque, segundo Kahneman e Tvsersky (1979, o tomador de decisão tende em manter o status quo.

A partir desta perspectiva de que o sistema emocional e psicológico governa uma determinada decisão e, portanto, sistematicamente varia de acordo com características relevantes do ambiente cultural, a literatura aponta que um ambiente de risco e incerteza promove reações relevantes nos indivíduos. No que diz respeito à influência da cultura sobre as preferências econômicas, segundo Apicella, Azevedo, Christakis e Fowler (2014), de acordo com os dados encontrados, fatores culturais e ambientais desempenham um papel chave na presença do efeito de dotação na tomada de decisão.

Embora a influência da cultura sobre as preferências de risco seja grande, nos resultados alcançados por Wang et al. (2016) e Rieger, Wang e Hens (2014), a característica fundamental é que os indivíduos têm aversão ao risco em ganhos e são propensos ao risco em perdas, como sugerido pela Teoria dos Prospectos de Tvsersky e Kahneman (1979), o que parece prevalecer globalmente. Isso demonstra que o ambiente de incerteza potencializa a propensão ao risco do indivíduo que tem aversão à perda.

Por consequência, o componente risco exerce influência na contabilidade gerencial, Sprinkle, Williamson e Upton (2008) investigaram a influência do comportamento de risco perante o desempenho do orçamento. Os autores ressaltam a importância da contabilidade gerencial nesse processo. Hall (2008) sinalizou que o sistema de medição de desempenho está ligado indiretamente com variáveis emocionais, que afetam a motivação dos gestores. Lima Filho e Bruni (2013) identificaram a presença de erros em julgamentos envolvendo situações relativas ao orçamento, em razão das heurísticas.

Pompian (2006) desenvolveu um trabalho sobre os conceitos de finanças comportamentais na gestão empresarial, fornecendo uma relação com vários vieses comportamentais (dentre eles, aversão à perda) que afetam os gestores na tomada de decisão sob risco.

Tais vieses, juntamente com a cultura, são explorados também na literatura sobre o enraizamento gerencial, que são as decisões que impactam a empresa e o desempenho organizacional (Ellili, 2012).

Portanto, os indícios direcionam para a relevância da compreensão dos vieses que podem afetar o processo de tomada de decisão dos indivíduos que atuam na área gerencial. Entretanto, há um espaço a ser explorado ao ponderar a potencialidade do contexto cultural neste processo.

\subsection{Conservadorismo Cultural e Contábil}

Tratando-se de aspectos culturais na Contabilidade, a maior parte dos pesquisadores utiliza o estudo de Gray (1988). O autor identificou os seguintes valores culturais relacionados à contabilidade: Profissionalismo (julgamento profissional individual livre) versus Controle Estatutário (cumprimento de aspectos legais); Uniformidade (práticas de contabilidade uniformes) versus Flexibilidade (práticas de acordo com a estrutura de cada entidade); Conservadorismo (postura mais prudente relacionada à incerteza de eventos futuros) versus Otimismo (postura para assumir riscos), e; Secretismo (restrição na divulgação das informações para quem está envolvido no negócio) versus Transparência (política de divulgação para os usuários externos). 
No Brasil, existem poucas pesquisas que abordam o efeito cultural na atividade contábil. Contudo, nos últimos anos, principalmente, alguns autores investigaram a relação de fatores culturais, sociais e econômicos com a decisão de adotar e emitir normas internacionais de contabilidade e nas práticas contábeis (Carvalho, Albuquerque, Quirós, \& Justino, 2015; Castro Neto, 1998; Castro Neto, Itoz, Lima, Pasqual, Bastos, \& Muller, 2006; Doupnik \& Riccio, 2006; Lima, 2016; Macêdo, Lopes, Silva, Ribeiro Filho, Pederneiras, \& Feitosa, 2010; Machado \& Nakao, 2014).

Para Dickhaut et al. (2010), o conservadorismo começou no início do século XV, antes da publicação do texto de Pacioli sobre as partidas dobradas, em que o tratamento pelo ativo deve ser pelo menor valor entre o custo e o valor de mercado. Os autores relacionam este entendimento à célebre frase: "reconhecer todas as perdas, mas não antecipar os ganhos".

Sob a ótica do mercado, os achados de Basu (1997) apontam que o resultado contábil, quando há más notícias, apresenta maior associação com retornos negativos da ação e, quando há boas notícias, associa-se a retornos positivos. Portanto, o viés conservador do ser humano está alinhado com o conservadorismo das práticas contábeis.

A cultura influencia os valores de um grupo, os quais atingem diretamente o comportamento nas decisões dos indivíduos. Nessa lógica, Kang et al. (2004) acreditam que a cultura impacta indiretamente o comportamento contábil como, por exemplo, as escolhas dos métodos contábeis. Os autores, ao analisar o resultado de suas pesquisas, identificaram que os gestores em ambientes culturais mais conservadores tendem a relatar estimativas mais baixas para os fluxos de caixa futuros. Em um estudo com operadores de contabilidade no Brasil, Lima (2016) identificou que o conservadorismo estava presente culturalmente.

O conservadorismo cultural, para Kang et al. (2004), desempenha um papel relativamente mais importante nas escolhas contábeis conservadoras dos gestores nos países code law do que nos países common law. Por exemplo, no Brasil (país com estrutura code law), Castro Neto et al. (2006) e Carvalho et al. (2015) concluíram em suas pesquisas que o país adotava práticas conservadoras.

Assim, a análise de Hofstede, segundo Kang et al. (2004), não discute conservadorismo diretamente, mas indica que os valores comumente associados podem ser encontrados em culturas com alta distância de poder (PDI) e de aversão à incerteza (UAI), baixo individualismo (IDV), masculinidade (MAS) e orientação de curto prazo (LTO). No entanto, quando se trata da relação entre práticas contábeis conservadoras e cultura, o estudo de Gray (1988) demonstra que há uma correlação quando são utilizadas as dimensões culturais de Hofstede, conforme a Tabela 3:

Tabela 3

Correlação entre culturas e valores contábeis conservadores

Dimensões culturais de Hofestde
Individualismo (IDV)
Masculinidade (MAS)
Aversão à incerteza (UAI)

Valores contábeis conservadores de Gray

Correlação negativa

Correlação negativa

Correlação positiva

Nota. Adaptado de Gray (1988).

Para Dickhaut et al. (2010), apesar da literatura atual não associar os princípios contábeis com as questões da neurociência, sinaliza que existe uma ligação paralela entre a contabilidade culturalmente evoluída e o comportamento do cérebro humano em uma situação de tomada de decisão. Segundo esses autores, a pesquisa baseada na atividade cerebral sugere um forte padrão de comportamento do cérebro 
consistente com o princípio de conservadorismo, pois o processo cognitivo do cérebro opera diferentemente a forma dos ganhos e das perdas.

Isso sugere que o conservadorismo, que está ligado principalmente ao princípio da prudência, possui raízes intrínsecas ao ser humano, que pode ser vista pelos valores culturais (Gray, 1988). Diante disso, aliado ao princípio da prudência, os contadores, quando estão sob o gerenciamento de informações contábeis, tendem a reconhecer mais antecipadamente as notícias de perdas do que as de ganhos. Isso pode ocorrer em razão da formação acadêmica e profissional da área contábil ser propensa a ser mais conservadora (Melo \& Silva, 2010).

\subsection{Hipóteses}

Até aqui, a fundamentação teórica, juntamente com resultados de outras pesquisas direcionam a construção das hipóteses. Rieger et al. (2014) estudaram o impacto cultural sob a preferência de risco no comportamento dos indivíduos em 53 países. Os resultados apontaram que o grau de aversão ao risco mostrou diferenças significativas entre os países. As atitudes de risco dependem não somente das condições econômicas, mas de fatores culturais, principalmente pelas dimensões Individualismo/Coletivismo (IDV) e Orientação de Curto / Longo Prazo (LTO). Wang et al. (2016) identificaram que o individualismo (IDV), percepção de distância do poder (PDI) e Masculinidade (MAS) aumentam o nível de aversão à perda significativamente.

A literatura indica que a cultura geralmente influencia o comportamento de um grupo específico de pessoas. Para Kang et al. (2004), escolhas contábeis entre métodos alternativos de evidenciação podem ser descritas como comportamento contábil discricionário dentro de subculturas de uma determinada economia. O mesmo autor, ao ressaltar as dimensões culturais de Hofstede, salienta que conservadorismo pode ser encontrado em culturas com alta distância de poder (PDI) e de aversão à incerteza (UAI), baixo individualismo (IDV) e masculinidade (MAS). Todavia, boa parte dos autores desconsideram a dimensão cultural (PDI), em razão da indicação proposta por Gray (1988), conforme a Tabela 3.

Nos trabalhos de Dickhaut (2009) e Dickhaut et al. (2010) nota-se o conservadorismo como uma característica implícita do ser humano, associada positivamente com a construção dos princípios e práticas contábeis. Portanto, os indivíduos que operam a contabilidade tendem a ser mais conservadores, primeiramente, pela característica inata e, em segundo, por valorizar mais as perdas pela influência conservadora do princípio da prudência. Além disso, Melo e Silva (2010) identificaram indícios que o profissional formado em Contabilidade possui maior aversão à perda. Com base no exposto, é possível conjecturar a seguinte hipótese:

Hipótese 1: O profissional com formação em Contabilidade, que atua na área gerencial, apresenta maior grau de aversão à perda.

No entanto, os valores culturais, neste estudo adotado pelo sociólogo Hofstede, provavelmente têm as implicações mais diretas nos comportamentos de escolha contábil dos gestores (Han, Kang, Salter, \& Yoo, 2010), em que o indivíduo formado em Contabilidade, que atua na área gerencial, pode realizar ações conservadoras, a partir de uma tendência de diferir o reconhecimento de ativos e itens que aumentam o lucro líquido (receitas, lucros, ganhos), além de uma tendência para acelerar o reconhecimento de passivos e itens que diminuem o lucro líquido (despesas, perdas) (Khlif, 2016).

Na Contabilidade Gerencial, Etemadi, Dilami, Bazaz e Parameswaran (2009) notaram que os sistemas da área e a participação do orçamento tiveram impacto na gestão de desempenho, tendo a relação 
com o menor nível de individualismo (IDV) e maior nível de masculinidade (MAS). Já a percepção de distância do poder (PDI) e a aversão à incerteza (UAI) tiveram impacto negativo na gestão de desempenho.

A orientação cultural do profissional da área gerencial pode influenciar o processo de tomada decisão em ambiente com risco de perda que pode ser relacionada com conservadorismo (Kahneman et al., 1991). Há indícios que um ambiente cultural com maior PDI (Kang, 2004; Wang et al., 2016) e UAI (Gray, 1988; Kang, 2004; Doupnik \& Riccio, 2006) se relaciona positivamente com o nível de aversão à perda, enquanto IDV e MAS demonstraram uma relação negativa com o nível de aversão à perda (Gray, 1988; Kang, 2004; Doupnik \& Riccio, 2006; Wang et al., 2016; Rieger et al., 2014). Portanto, formulou-se a seguinte hipótese:

Hipótese 2: Quanto maior o nível de dimensão cultural PDI e UAI, maior o grau de aversão à perda dos indivíduos formados em contabilidade.

Hipótese 3: Quanto menor o nível de dimensão cultural IDV e MAS, maior o grau de aversão à perda dos indivíduos formados em contabilidade.

\section{METODOLOGIA}

A pesquisa foi operacionalizada por meio de uma survey para coleta de dados, baseada no protocolo desenvolvido por Wang et al. (2016). Os questionários foram aplicados aos estudantes de pósgraduação lato sensu nas áreas que envolvem contabilidade na área gerencial na cidade de Salvador. A composição desta amostra fundamenta-se na estrutura mais heterogênea, além da probabilidade de estar atuando no mercado (área gerencial) ser maior em relação a estudantes de graduação, pois as perguntas do questionário, em sua maioria, são direcionadas ao ambiente de trabalho.

O questionário utilizado "Values Survey Module 1994" divide-se em três partes: informações sobre risco, dimensões culturais e dados demográficos. A aplicação foi dividida entre a divulgação por e-mail e in loco. Todavia, a última representou 93\% das repostas obtidas.

Como intuito de estruturar o desenho da pesquisa, foi utilizado o modelo de Libby, Bloomfield e Nelson (2002), conforme a Figura 1, com a finalidade de sustentar a fundamentação da relação entre as variáveis sob a ótica da validade externa e interna.

Com relação à validade externa (link 1) do libby box, o apoio conceitual principal da variável independente baseia-se nas dimensões culturais propostas por Hofstede (2001), que possuem, na literatura, indícios de influenciar o comportamento de aversão à perda dos indivíduos (Tvsersky \& Kahneman, 1992). O link 2 refere-se às variáveis operacionais exploradas conceitualmente por Hofstede (2001), por meio de quatro dimensões culturais. Já a variável conceitual dependente fundamenta-se no trabalho sobre aversão à perda de Tvsersky e Kahneman (1992). A operacionalização dessa varável (link 3) segue o protocolo elaborado por Wang et al. (2016), por meio de dois jogos de loteria (Figura 3). O link 4 é o meio operacional principal, por meio das dimensões culturais utilizadas, que são independentes, com o intuito de verificar se exerce influência no nível de aversão à perda adaptado por Wang et al. (2016). Para controlar as variáveis dependentes (link 5), foram considerados o perfil profissional, gênero e idade pela capacidade de influenciar o nível de aversão à perda (Melo \& Silva, 2010; Wang et al., 2016).

Figura 1 


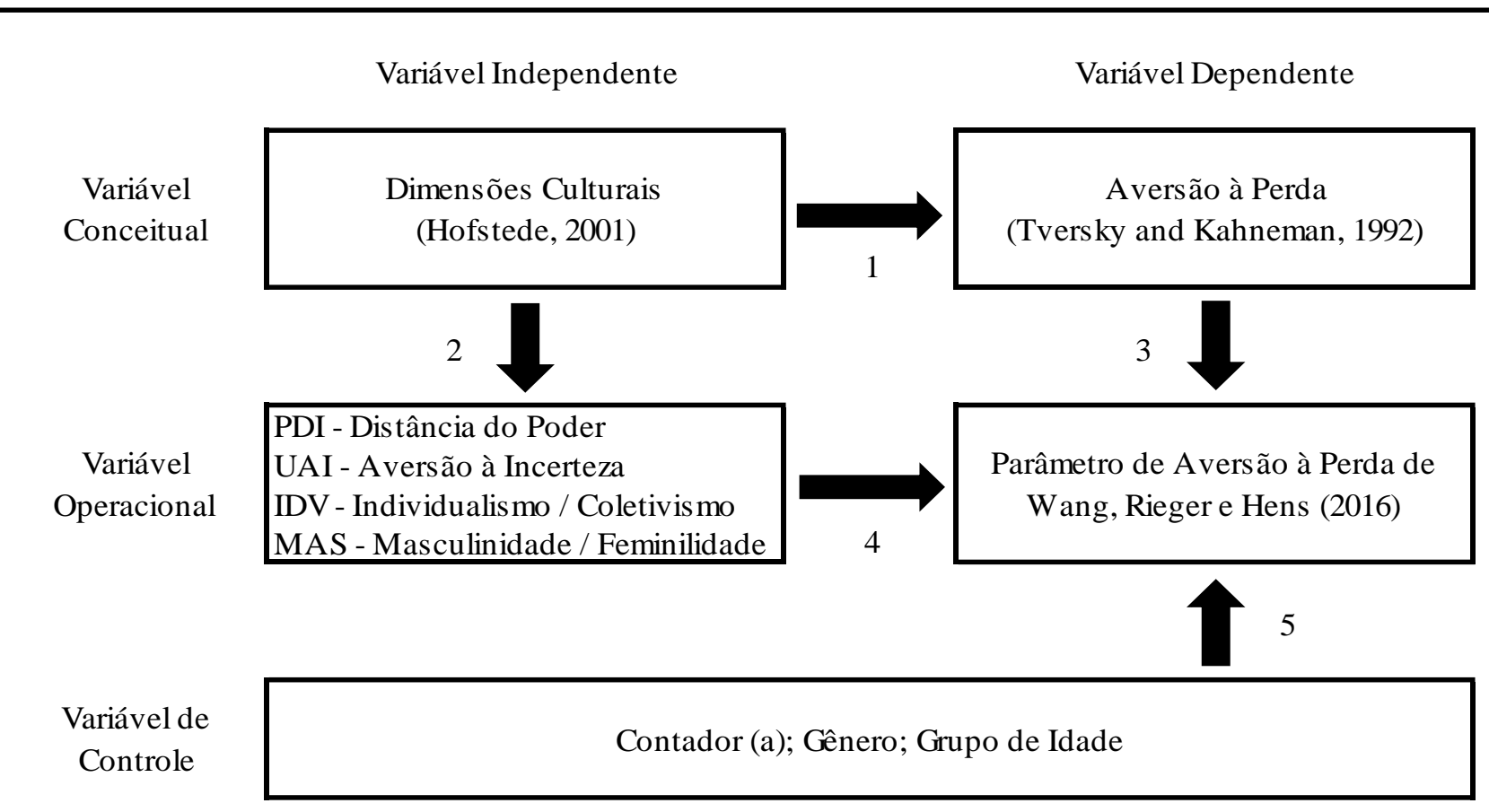

Quanto às informações sobre perda e risco, Wang et al. (2016) e Rieger et al. (2014), respectivamente, basearam-se na Teoria dos Prospectos, desenvolvida por Kahneman e Tversky (1979). O problema, conforme a Figura 2, mensura a aversão à perda por meio de um jogo. Neste jogo, os jogadores têm $50 \%$ de chance de ganhar ou perder dinheiro. Para isso, é necessário que se indique a quantidade mínima de dinheiro nos jogos A e B que estaria disposto como exigência para participar destes jogos.

Para medir a aversão à perda dos participantes, foi utilizado o parâmetro " $\theta$ " de Tversky e Kahneman (1992). Se $\theta=1$, significa que há neutralidade na perda. Se $\theta>1$, significa aversão à perda. Logo depois, verificou-se o parâmetro para cada jogo através da seguinte equação:

$$
\theta a=\frac{x}{25} \text { e } \quad \theta b=\frac{X}{100}
$$

Em que $\theta \mathrm{a}$ e $\theta \mathrm{b}$ são parâmetros de aversão à perda pela menor participação (R $\$ 25,00)$ e maior participação (RS 100,00), respectivamente. Na sequência, foi verificada a possibilidade de utilizar a média de aversão à perda, por meio de análise fatorial exploratória, conforme a Tabela 4. Nesta análise fatorial, dividiu-se a amostra em relação à formação em Contabilidade, com o intuito de aplicar o mesmo parâmetro (média) para ambos os grupos nos testes estatísticos vistos na próxima seção.

Figura 2 
Jogo lotérico para identificar aversão à perda

\begin{tabular}{ccc}
\hline A & $\begin{array}{l}50 \% \text { chance } \\
50 \% \text { chance }\end{array}$ & $\begin{array}{l}\text { perda de } \$ 25 \\
\text { ganho de } \$ \mathbf{X}\end{array}$ \\
\hline \multicolumn{4}{c}{ O valor mínimo de $\$ \mathbf{X}$ para tornar o jogo aceitável } \\
& & \\
\hline \multirow{2}{*}{ B } & $50 \%$ chance & perda de $\$ 100$ \\
& $50 \%$ chance & ganho de $\$ \mathbf{Y}$ \\
\hline
\end{tabular}

O valor mínimo de \$ Y para tornar o jogo aceitável

A rotação fatorial foi por meio do método ortogonal de varimax. O resultado apontou que o modelo fatorial atende os requisitos mínimos. Para Hair, Anderson, Tatham e Black (2005), o KMO de 0,500, a variância acumulada superior a 60\% e o índice de significância de BTS menor que 5\% sinalizam níveis aceitáveis diante dos grupos de profissionais que atuam na área gerencial formados em Contabilidade e de profissionais que atuam na área gerencial não formados em Contabilidade.

Portanto, a utilização da média de aversão à perda foi aceita. Muito embora, a média do jogo A foi maior em relação ao jogo B, construindo assim, uma lógica inversa perante a literatura, uma vez que Tversky e Kahneman (1992) indicam que o nível de aversão aumenta ao passo do valor de perda.

Tabela 4

Redução de fator de aversão à perda

\begin{tabular}{c|c|c|c|c|c}
\hline Grupo & $\begin{array}{c}\text { Component } \\
\text { es }\end{array}$ & $\begin{array}{c}\text { Único } \\
\text { Component } \\
\text { e Principal }\end{array}$ & $\begin{array}{c}\text { \% da } \\
\text { variância }\end{array}$ & $\begin{array}{c}\text { KM } \\
\text { O }\end{array}$ & $\begin{array}{c}\text { BTS } \\
\text { (sig) }\end{array}$ \\
\hline $\begin{array}{c}\text { Profissionais de gestão com formação em } \\
\text { Contabilidade }\end{array}$ & $\begin{array}{c}\text { AversãoA } \\
\text { AversãoB }\end{array}$ & 1,293 & 64,63 & 0,5 & 0,019 \\
\hline $\begin{array}{c}\text { Profissionais de gestão sem formação em } \\
\text { Contabilidade }\end{array}$ & $\begin{array}{c}\text { AversãoA } \\
\text { AversãoB }\end{array}$ & 1,319 & 65,93 & 0,5 & 0,020 \\
\hline
\end{tabular}

Nota. Dados da pesquisa. KMO = Kaiser-Meyer-Olkin; BTS = Bartlett's Test of Sphericity.

A mensuração das dimensões culturais e os dados demográficos foram por meio do questionário indicado por Hofstede (2001), titulado: "VALUES SURVEY MODULE 1994". Foram selecionadas as dimensões: PDI, UAI, IDV e MAS, seguindo o protocolo de Wang et al. (2016). Contudo, foi retirada a pergunta referente à escolaridade, uma vez que os respondentes são alunos de pós-graduação. Além disso, foram adicionadas perguntas sobre a formação acadêmica e área de atuação profissional.

Para verificar se as dimensões culturais impactam no comportamento dos contadores foram elaborados dois modelos de regressão linear pelo Método dos Mínimos Quadrados Ordinários (MQO), conforme equações 1 e 2 .

$$
\begin{aligned}
& \text { LgAVS }=\beta+\beta P D I+\beta U A I-\beta I D V-\beta M A S+\beta C O N T+\beta S E X O+\beta I D A D E+\varepsilon \\
& \text { LgAVS }=\beta+\beta P D I c o n t+\beta U A I c o n t-\beta I D V \text { cont }-\beta M A S c o n t+\beta S E X O+\beta I D A D E+\varepsilon
\end{aligned}
$$




\section{Em que:}

LgAVS = variável dependente que representa o logaritmo da média de aversão à perda

$\beta=$ constante da regressão

$\beta \mathrm{PDI}=$ variável independente que representa Distância do Poder

$\beta I D V=$ variável independente que representa Individualismo / Coletivismo

$\beta \mathrm{MAS}=$ variável independente que representa Masculinidade / Feminilidade

$\beta U A I=$ variável independente que representa Aversão à Incerteza

$\beta$ PDIcont $=$ variável independente que representa o produto de $\beta$ PDI $x \beta C O N T$

$\beta I D V$ cont $=$ variável independente que representa o produto de $\beta$ IDV $\times \beta C O N T$

$\beta$ MAScont $=$ variável independente que representa o produto de $\beta$ MAS $\times \beta C O N T$

$\beta$ UAIcont $=$ variável independente que representa o produto de $\beta$ UAI $x \beta C O N T$

$\beta C O N T$ = variável de controle dummy que representa a formação acadêmica em contabilidade

$\beta S E X O$ = variável de controle dummy que representa o sexo do indivíduo

$\beta I D A D E=$ variável de controle que representa o grupo de idade do indivíduo

$\varepsilon=$ termo de erro da regressão

Nota-se que a variável dependente foi transformada em valores logarítmicos. Essa medida melhorou a qualidade dos dados, tendo em vista os outliers encontrados, conforme a Figura 3.

\section{Figura 3}

Diagramas de caixa da variável dependente original (à esquerda) e a sua transformação em valores logarítmicos (à direita)
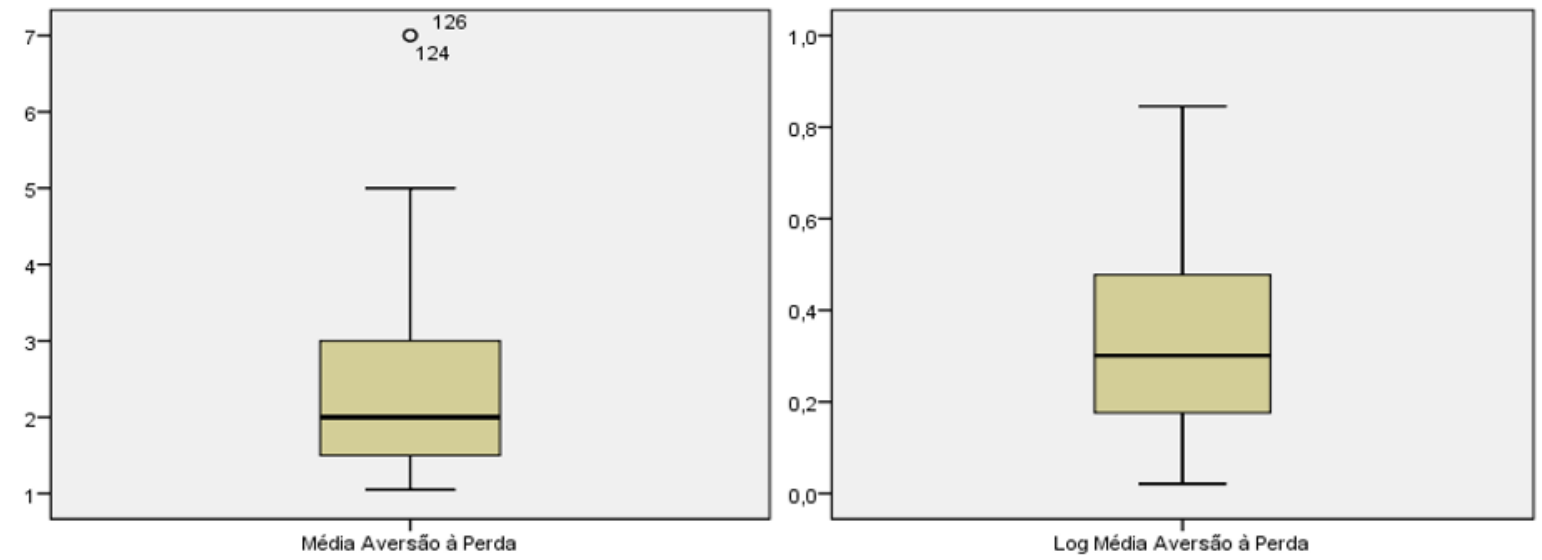

Nota. Dados da pesquisa.

\section{ANÁLISE DOS RESULTADOS}

O questionário foi aplicado nos cursos de pós-graduação, em áreas relacionadas à gestão empresarial na cidade de Salvador. Foram entregues 147 questionários, obtendo um retorno de 126 devidamente preenchidos. Após iniciar o tratamento estatístico, nove questionários foram excluídos, pois os respondentes não demonstraram aversão à perda $(\theta \leq 1)$, de acordo com Tversky e Kahneman (1992) e Wang et al. (2016). Ao final, 117 questionários foram considerados válidos, conforme a Tabela 5.

Tabela 5 
Característica da amostra

\begin{tabular}{|c|c|c|c|c|c|c|}
\hline & \multicolumn{3}{|c|}{$\begin{array}{c}\text { Profissionais da área gerencial formados em } \\
\text { Contabilidade }\end{array}$} & \multicolumn{3}{|c|}{$\begin{array}{l}\text { Profissionais da área gerencial não formados em } \\
\text { Contabilidade }\end{array}$} \\
\hline & Frequência & $\%$ & \% Cumulativo & Frequência & $\%$ & \% Cumulativo \\
\hline $\begin{array}{l}\text { Entre } 20-24 \\
\quad \text { anos }\end{array}$ & 6 & 9,38 & 9,38 & 11 & 20,75 & 20,75 \\
\hline $\begin{array}{l}\text { Entre } 25-29 \\
\quad \text { anos }\end{array}$ & 27 & 42,19 & 51,56 & 17 & 32,08 & 52,83 \\
\hline $\begin{array}{l}\text { Entre } 30-34 \\
\quad \text { anos }\end{array}$ & 16 & 25,00 & 76,56 & 12 & 22,64 & 75,47 \\
\hline $\begin{array}{l}\text { Entre } 35 \text { - } 39 \\
\quad \text { anos }\end{array}$ & 11 & 17,19 & 93,75 & 4 & 7,55 & 83,02 \\
\hline $\begin{array}{l}\text { Entre } 40 \text { - } 49 \\
\quad \text { anos }\end{array}$ & 2 & 3,13 & 96,88 & 4 & 7,55 & 90,57 \\
\hline $\begin{array}{l}\text { Entre } 50-59 \\
\quad \text { anos }\end{array}$ & 2 & 3,13 & 100 & 3 & & 96,23 \\
\hline $\begin{array}{l}\text { Mais de } 60 \\
\text { anos }\end{array}$ & & & & 2 & 3,77 & \\
\hline Total & 64 & 100 & & 53 & 100 & \\
\hline Homem & 33 & 51,56 & & 29 & 54,72 & \\
\hline Mulher & 31 & 48,44 & & 24 & 45,28 & \\
\hline Total & 64 & 100 & & 53 & 100 & \\
\hline
\end{tabular}

Nota. Dados da pesquisa.

De acordo com a Tabela 5, dos 117 respondentes que atuam na área gerencial, 64 possuem formação em Contabilidade, sendo os demais formados em outras áreas. Em ralação à variável gênero, 33 homens e 31 mulheres totalizam aqueles formados em Contabilidade. Dos outros 53 com formação em outras áreas, 29 são homens e 24 mulheres.

Em relação à idade, $51,56 \%$ da amostra formada em contabilidade possui menos de 30 anos. Dado similar em relação aos indivíduos com formação em outras áreas $(52,83 \%)$. Portanto, metade da amostra possui uma idade inferior a 30 anos $(52,14 \%)$.

Diante da Tabela 5, a amostra apresentou uma característica equilibrada do grupo de profissionais da área gerencial que são formados em Contabilidade em relação aos não formados em Contabilidade, no que tange às variáveis de gênero e idade. Esse cenário mais homogêneo promove uma estrutura estatística mais adequada na análise dos resultados.

Tabela 6

Pressupostos dos Modelos de Regressão

\begin{tabular}{cccc}
\hline Teste & Método de Verificação & Modelo 1 & Modelo 2 \\
\hline Erro de especificação & Reset de Ramsey (sig) & 0,827 & 0,367 \\
Normalidade & Shapiro-wilk (sig) & 0,004 & 0,000 \\
Heteroscedasticidade & Breusch-Pagan / Cook-weisberg (sig) & 0,843 & 0,838 \\
Multicolinearidade & Tolerance/VIF & Variáveis em torno de 1,0 \\
\hline
\end{tabular}

Fonte: Dados da pesquisa.

Para Hair et al. (2005), apesar da proporção mínima ser de cinco observações para cada variável, o ideal deve estar entre 15 e 20 observações. Portanto, o tamanho da amostra está dentro do parâmetro 
adequado. No entanto, para garantir a robustez do modelo foram testados os pressupostos dos modelos de regressão.

Conforme apresentado na Tabela 6, os modelos estão bem especificados, como também existem variáveis omitidas. O teste referente à distribuição normal dos resíduos indicou que ambos os modelos de regressão apresentaram problemas, pois o nível de significância foi menor que 5\%. Neste caso, foi utilizada a técnica de regressão não paramétrica bootstrap. Quando a distribuição dos erros é desconhecida e não normal, este método permite estimar os parâmetros (Chernick \& Labudde, 2011). Nos dois modelos foram estabelecidas 1.000 reamostragens com intervalo de confiança de $95 \%$.

Em seguida foi aplicado o teste de heteroscedasticidade, para identificar se os dados regredidos estão concentrados em torno da reta. $\mathrm{O}$ resultado de ambos os modelos demonstrou um comportamento homogêneo dos dados. Os modelos não apresentaram problema de multicolinearidade, portanto, não há forte correlação entre as variáveis independentes, já que os valores do test VIF e Tolerance estão em torno de 1.

O primeiro modelo de regressão, conforme apresentado na Tabela 7, foi destinado a identificar o impacto das dimensões culturais, considerando todas as demais variáveis, nas decisões de todos os indivíduos em relação à aversão à perda.

\section{Tabela 7}

Regressão Multivariada do modelo 1

\begin{tabular}{|c|c|c|c|c|c|c|}
\hline \multirow{2}{*}{ Variáveis } & & \multirow{2}{*}{ Coeficiente } & \multirow{2}{*}{ Erro Padrão } & \multirow{2}{*}{ Sig, (bicaudal) } & \multicolumn{2}{|c|}{ Invervalo de Confiança } \\
\hline & & & & & Menor & Maior \\
\hline PDI & & 0,0003 & 0,0021 & 0,8881 & $-0,0039$ & 0,0045 \\
\hline UAI & & 0,0024 & 0,0018 & 0,1798 & $-0,0010$ & 0,0061 \\
\hline IDV & & 0,0031 & 0,0023 & 0,1728 & $-0,0018$ & 0,0076 \\
\hline MAS & & 0,0000 & 0,0010 & 0,9740 & $-0,0020$ & 0,0019 \\
\hline Formação Acadêmica & & 0,5642 & 0,2073 & 0,0070 & 0,1753 & 0,9728 \\
\hline Gênero & & 0,5001 & 0,2188 & $\mathbf{0 , 0 3 4 0}$ & $\mathbf{0 , 0 5 5 4}$ & $\mathbf{0 , 9 7 2 0}$ \\
\hline Idade & & 0,0190 & 0,0768 & 0,8172 & $-0,1181$ & 0,1660 \\
\hline (Constant) & & 1,3165 & 0,3865 & 0,0020 & 0,5875 & 2,0936 \\
\hline $\mathrm{F}$ & 2,47 & 0,0214 & & & & \\
\hline $\mathrm{N}$ & 117 & & & & & \\
\hline $\mathrm{R}^{2}(\%)$ & 13,7 & & & & & \\
\hline
\end{tabular}

Nota. Dados da pesquisa. Variável dependente = Log da média de aversão à perda. Formação acadêmica é dummy (1 para formação em contabilidade e 0 para as demais). Gênero é dummy (1 para masculino e 0 para feminino). Idade é formada por sete grupos, conforme proposto por Hofstede. PDI = Distância do Poder; UAI = Aversão à Incerteza; IDV = Individualismo / Coletivismo; MAS = Masculinidade / Feminilidade; LTO = Orientação de Curto / Longo Prazo.

A Tabela 7 apresenta o resultado da regressão multivariada do primeiro modelo. O teste $\mathrm{F}$ trata a igualdade da média quadrática da regressão sobre a média quadrática dos resíduos. O resultado (sig < 0,05) permite identificar que há pelo menos um beta diferente de zero, ou seja, existe relação linear da variável dependente com pelo menos uma variável independente. $\mathrm{O} \mathrm{R}^{2}$ do primeiro modelo foi de 0,137 , indicando que $13,7 \%$ da variância das variáveis independentes possui poder de explicar o nível de aversão à perda.

O primeiro modelo de regressão apontou que nenhuma dimensão cultural influencia o comportamento de aversão à perda dos indivíduos em geral. A variável formação acadêmica apontou 
significativamente impacto na variável dependente. $\mathrm{O} \beta=0,5642$, com sinal positivo, indica uma tendência que os profissionais da área gerencial formados em contabilidade possuem maior nível de aversão à perda. Este resultado corrobora a primeira hipótese, em que a formação em Contabilidade reflete uma vocação mais conservadora, convergindo com os estudos de Dickhaut (2009) e Dickhaut et al. (2010).

A variável gênero apontou impacto significante $(\operatorname{sig}=0,034)$, indicando que os homens são mais propensos a serem avessos à perda $(\beta$ positivo $=0,5001)$, divergindo dos achados de Wang et al. (2016). A outra variável independente (idade) não demonstrou capacidade de influenciar a variável dependente (média de aversão à perda). Todos os resultados do bootstrap dos dois modelos estão validados ao considerar também o intervalo de confiança.

Com relação às dimensões culturais, os resultados não indicaram qualquer influência no nível de aversão à perda.

O próximo modelo de regressão, conforme a Tabela 8, apresenta a influência das variáveis culturais, como moderadoras, nos profissionais da área gerencial, aprofundando a investigação daqueles com formação em Contabilidade.

\section{Tabela 8}

Regressão Multivariada do modelo 2

\begin{tabular}{|c|c|c|c|c|c|c|}
\hline \multirow{2}{*}{ Variáveis } & & \multirow{2}{*}{ Coeficiente } & \multirow{2}{*}{ Erro Padrão } & \multirow{2}{*}{ Sig, (bicaudal) } & \multicolumn{2}{|c|}{ Invervalo de Confiança } \\
\hline & & & & & Menor & Maior \\
\hline PDIcont & & 0,0005 & 0,0028 & 0,8521 & $-0,0046$ & 0,0065 \\
\hline UAIcont & & 0,0037 & 0,0016 & $\mathbf{0 , 0 2 8 0}$ & 0,0005 & 0,0068 \\
\hline IDVcont & & 0,0019 & 0,0021 & 0,3696 & $-0,0023$ & 0,0063 \\
\hline MAScont & & $-0,0037$ & 0,0015 & $\mathbf{0 , 0 1 3 0}$ & $-0,0066$ & $-0,0008$ \\
\hline Gênero & & 0,3697 & 0,1895 & 0,0639 & 0,0049 & 0,7621 \\
\hline Idade & & $-0,0251$ & 0,0570 & 0,6464 & $-0,1425$ & 0,0840 \\
\hline (Constant) & & 1,9730 & 0,2050 & 0,0010 & 1,5940 & 2,3967 \\
\hline $\mathrm{F}$ & 3,459 & 0,0036 & & & & \\
\hline $\mathrm{N}$ & 64 & & & & & \\
\hline $\mathrm{R}^{2}(\%)$ & 16 & & & & & \\
\hline
\end{tabular}

Nota. Dados da pesquisa. Variável dependente $=$ Log da média de aversão à perda. Gênero é dummy (1 para masculino e 0 para feminino). Idade é formada por sete grupos, conforme proposto por Hofstede. PDIcont = resultado do produto entre PDI e Formação acadêmica (dummy); UAIcont = resultado do produto entre UAI e Formação acadêmica (dummy); IDVcont = resultado do produto entre IDV e Formação acadêmica (dummy); MAScont = resultado do produto entre MAS e Formação acadêmica (dummy).

O segundo modelo de regressão apresenta um $\mathrm{R}^{2}=0,16$, indicando que $16 \%$ da variância das variáveis independentes e de controle possui poder de explicar a variável dependente. $\mathrm{O}$ resultado do teste $\mathrm{F}(\mathrm{sig}=0,0036)$, seguindo o modelo anterior, permite a inferência que há pelo menos um beta diferente de zero, ou seja, existe relação linear da variável dependente com pelo menos uma variável independente.

Neste modelo, cuja intenção é a interação entre o profissional da área gerencial com formação acadêmica e as dimensões culturais, apontou que algumas dimensões culturais podem exercer influência na manutenção da característica conservadora dos profissionais formados em Contabilidade que atuam na área gerencial, comparação aos não formados em contabilidade. 
Sobre a H2, que buscou identificar o impacto do PDI e UAI no nível de aversão à perda dos profissionais da área gerencial com formação em Contabilidade, notou-se que foi aceita parcialmente. Ressalta-se que houve também alinhamento parcial dos resultados com a hipótese 3, em que buscou identificar a influência do IDV e MAS.

Sobre a segunda hipótese, os resultados apontaram que UAI demonstrou capacidade de influenciar o nível de aversão à perda dos profissionais da área gerencial com formação em Contabilidade, já que o sinal do coeficiente é positivo $(\beta=0,0037)$ e o nível significância é de 0,0280 (sig < 5\%). De acordo com sinal positivo da variável UAI, permite-se associar maior grau de aversão à incerteza, ao passo do crescimento no nível de aversão à perda destes profissionais. Portanto, tal hipótese é aceita, de acordo com os achados de Gray (1988), Kang et al. (2004), Doupnik e Riccio (2006) e proposição de Wang et al. (2016).

O resultado da regressão apontou também que a dimensão cultural MAS impacta o nível de aversão à perda de forma significativa ( $\mathrm{sig}=0,013)$, uma vez que o profissional de gestão é formado em Contabilidade. $\mathrm{O}$ sinal negativo da constante de MAS $(\beta=-0,0037)$ indica que quanto maior grau de aversão à perda, menor é a percepção masculina dos profissionais com formação na área contábil. Esse resultado valida a hipótese H3, seguindo os achados de Gray (1988), Kang (2004), Doupnik e Riccio (2006), Wang et al. (2016) e Rieger et al. (2014).

Com relação às variáveis de controle (gênero e idade), a variável idade não apresentou indícios estatísticos significativos. Já a variável gênero demonstrou evidência (sig = 0,0639) de impacto sobre a variável dependente. Tais resultados foram mantidos conforme visto na Tabela 8. Esse resultado estimulou uma investigação mais detalhada, de acordo com a Tabela 9, que trata do teste não paramétrico de duas amostras independentes (gênero), divididas por grupo (profissionais da área gerencial formados em Contabilidade e profissionais da área gerencial não formados em Contabilidade).

Tabela 9 - Teste não paramétrico de duas amostras independentes para gênero

\begin{tabular}{|c|c|c|c|c|c|c|c|c|c|}
\hline \multirow{2}{*}{\multicolumn{2}{|c|}{$\begin{array}{l}\text { Variáveis de Teste = } \\
\text { Gênero }\end{array}$}} & \multicolumn{4}{|c|}{$\begin{array}{l}\text { Profissionais da área gerencial } \\
\text { formados em Contabilidade }\end{array}$} & \multicolumn{4}{|c|}{$\begin{array}{l}\text { Profissionais da área gerencial não } \\
\text { formados em Contabilidade }\end{array}$} \\
\hline & & $\mathbf{N}$ & Média & $\mathbf{U}$ & Sig. & $\mathbf{N}$ & Média & $\mathbf{U}$ & Sig. \\
\hline \multirow{2}{*}{$\begin{array}{l}\text { Log Média } \\
\text { Aversão à } \\
\text { Perda }\end{array}$} & Feminino & 33 & 28,89 & \multirow{2}{*}{392,5} & \multirow{2}{*}{0,107} & 29 & 25,41 & \multirow{2}{*}{302,5} & \multirow{2}{*}{0,407} \\
\hline & Masculino & 31 & 36,34 & & & 24 & 28,92 & & \\
\hline \multirow{2}{*}{ PDI } & Feminino & 33 & 32,35 & \multirow{2}{*}{506,5} & \multirow{2}{*}{0,946} & 29 & 31,59 & \multirow{2}{*}{215,0} & \multirow{2}{*}{0,170} \\
\hline & Masculino & 31 & 32,66 & & & 24 & 21,46 & & \\
\hline \multirow{2}{*}{ UAI } & Feminino & 33 & 33,97 & \multirow{2}{*}{463,0} & \multirow{2}{*}{0,514} & 29 & 29,10 & \multirow{2}{*}{287,0} & \multirow{2}{*}{0,275} \\
\hline & Masculino & 31 & 30,94 & & & 24 & 24,46 & & \\
\hline \multirow{2}{*}{ IDV } & Feminino & 33 & 36,12 & \multirow{2}{*}{392,0} & \multirow{2}{*}{0,107} & 29 & 29,71 & \multirow{2}{*}{269,5} & \multirow{2}{*}{0,159} \\
\hline & Masculino & 31 & 28,65 & & & 24 & 23,73 & & \\
\hline \multirow{2}{*}{ MAS } & Feminino & 33 & 28,82 & \multirow{2}{*}{390,0} & \multirow{2}{*}{0,101} & 29 & 26,60 & \multirow{2}{*}{336,5} & \multirow{2}{*}{0,837} \\
\hline & Masculino & 31 & 36,42 & & & 24 & 27,48 & & \\
\hline
\end{tabular}

Nota. Dados da pesquisa. Variáveis de teste: Log da média de aversão à perda. Gênero é dummy (1 para masculino e 0 para feminino). PDI = Distância do Poder; UAI = Aversão à Incerteza; IDV = Individualismo / Coletivismo; MAS = Masculinidade / Feminilidade; LTO = Orientação de Curto / Longo Prazo. U = Estatística não paramétrica de Mann-Whitney.

A Tabela 9 apresenta resultados do teste não paramétrico das médias simples entre os gêneros, divididas pelo grupo de profissionais da área gerencial com formação em Contabilidade e com 
profissionais da área gerencial formados em outras áreas. Os dois modelos de regressão apontaram evidências do impacto do gênero masculino. Todavia, o resultado da comparação de médias entre os gêneros não foi significante (sig > 0,05), em relação à média de aversão à perda, considerando os dois grupos de profissionais de gestão (com e sem formação em Contabilidade). Portanto, o gênero masculino dos profissionais de gestão tende a ser mais avesso à perda, independentemente da formação acadêmica.

Adicionalmente, buscou-se verificar a existência de diferenças entre os comportamentos de homens e mulheres, perante às dimensões culturais. $\mathrm{O}$ resultado apontou que não há influência significativa dessa variável sobre os aspectos da cultural local.

Por fim, a Tabela 10 apresenta um resumo da confrontação das hipóteses e os achados:

\section{Tabela 10}

Resumo das respostas às hipóteses

\begin{tabular}{cccc}
\hline Hipótese & Esperado & Resultado & Conclusão \\
H1 (Formação em Ciências Contábeis) & + aversão à perda & + aversão à perda & Hipótese aceita \\
H2 (Valor cultural PDI e UAI) & + aversão à perda & $\begin{array}{c}\text { - aversão à perda (PDI) } \\
+ \text { aversão à perda (UAI)* }\end{array}$ & Hipótese parcialmente aceita \\
H3 (Valor cultural IDV e MAS) & - aversão à perda & $\begin{array}{c}\text { + aversão à perda (IDV) } \\
\text { - aversão à perda (MAS)* }\end{array}$ & Hipótese parcialmente aceita \\
\hline
\end{tabular}

Nota. Dados da pesquisa. *estatisticamente significante.

\section{CONCLUSÃO}

Estudos sobre o comportamento humano nas decisões sob cenários de risco vêm crescendo nas últimas décadas, principalmente após a publicação dos trabalhos de Kahneman e Tversky, sobretudo quando o indivíduo considera o risco em ambiente de perda. Nota-se que o sentimento da dor de uma perda é mais agudo do que o prazer de um ganho equivalente (Thaler, 2001). Todavia, essa questão hedônica pode atingir de maneira diferente o processo decisório dos indivíduos de diferentes culturas.

Este estudo é justificado pela percepção da lacuna de pesquisas comportamentais, considerando dimensões culturais, principalmente sob um ambiente de contabilidade gerencial, em que os profissionais podem ter um comportamento conservador devido à formação acadêmica. Tal comportamento é um dos vetores responsáveis para influenciar as decisões de risco dos indivíduos. Entretanto, há indícios que a cultura possui certa influência nesse processo.

$\mathrm{O}$ artigo de Wang et al. (2016) serviu de referência para o desenvolvimento da pesquisa. O questionário foi aplicado aos estudantes que estão cursando pós-graduação lato sensu em áreas ligadas à gestão, razão pela qual a estrutura de perguntas é vinculada, em maior parte, à percepção de um indivíduo em um ambiente de trabalho. Portanto, a probabilidade de alunos de pós-graduação estarem trabalhando ou que já trabalharam é maior em relação aos estudantes de graduação. De fato isso foi confirmado, em razão da totalidade dos respondentes atuarem na área gerencial.

Foi utilizado o questionário indicado por Hofstede (2001): “Values Survey Module 1994”. Sua informação foi dividida em três partes: informações sobre risco, dimensões culturais e dados sociodemográficos. Com relação às informações sobre o risco, os autores basearam-se na Teoria dos Prospectos, desenvolvida por Kahneman e Tversky (1979). Para mensurar as dimensões culturais e os dados demográficos foi utilizado o questionário indicado por Hofstede (2001). 
No contexto generalizado, grande parte dos participantes desta pesquisa exigiu um ganho superior ao valor da perda. Nesta pesquisa, o objetivo central foi observar se a cultura, elemento extrínseco, é capaz de acentuar ou minimizar o status quo em um ambiente de perda dos profissionais da área gerencial, sobretudo com formação em Contabilidade.

Os resultados apontaram que a característica biológica do ser conservador dos profissionais da área gerencial com formação em Contabilidade foi mantida. Notou-se que a formação em Contabilidade promove uma percepção de maior aversão à perda (Basu, 1997; Dickhaut, 2009; Dickhaut et al., 2010).

Os achados indicam que os valores culturais não orientam o nível de aversão à perda de todos os indivíduos. Contudo, o valor cultural "Aversão à Incerteza" (UAI), em que os indivíduos agem mais com a perspectiva incerta do futuro, são mais avessos à perda. O valor cultural "Masculina x Feminina" (MAS), em que os respondentes com características culturais menos masculina evidenciam maior aversão à perda, demonstraram influência nos profissionais da área gerencial com formação em Contabilidade. Esses achados corroboram Wang et al. (2016) e Rieger et al. (2014, no sentido do impacto da cultura no nível de aversão à perda; com Gray (1988), Doupnik e Riccio (2006), Kang (2004), ao considerar os aspectos conservadores das práticas contábeis; e Etemadi et al. (2009), pelo alinhamento do impacto das dimensões culturais nas práticas da Contabilidade Gerencial.

Tais resultados promovem uma reflexão, em que a influência cultural, o risco e a incerteza, sob um ambiente de perda, resgatam o viés emocional dos profissionais da área gerencial formados em contabilidade, que podem ser influenciados pelos princípios e pelas normas e práticas com tendências mais conservadoras. Especificamente, colabora para uma compreensão de que sistemas de contabilidade gerencial iguais na mesma entidade podem provocar nos contadores(as) decisões diferentes e disfuncionais em razão do contexto cultural (Tsui, 2011; Busco \& Scapen, 2011).

Adicionalmente, os resultados indicaram que o gênero masculino tende a ser mais avesso à perda, independentemente do espectro cultural, divergindo dos achados Wang et al. (2016). Em relação à idade, esse aspecto não demonstrou influenciar o comportamento avesso à perda, mesmo considerando as dimensões culturais.

É preciso haver mais pesquisas sobre esta temática, com o intuito de fortalecer debates sobre o impacto nas práticas contábeis. Para Baskerville (2003), o uso das dimensões culturais de Hofstede por estudiosos de contabilidade levanta mais problemas do que resolve. O mesmo autor sugere abordagens alternativas para a investigação sobre as diferenças nacionais no que tange à contabilidade. Este estudo buscou avançar este ponto, em que Wang et al. (2016) não considerou profissionais que atuam na área gerencial e/ou com formação em Contabilidade. Portanto, estes achados podem contribuir para futuras pesquisas considerarem o profissional formado em Contabilidade como variável importante no processo de tomada de decisão em ambiente de risco.

Com relação à limitação da pesquisa, pela razão do público-alvo ser alunos de pós-graduação lato sensu na área de contabilidade, o universo amostral dificultou, quantitativamente, a captação de respondentes. Como também, a amplitude geográfica é a indicação mais utilizada na comparação dos aspectos culturais. Essas observações, em tempo, servem como sugestão de trabalhos futuros, pois existe

uma lacuna em pesquisas comportamentais, sobretudo na área contábil, que pondere aspectos culturais locais.

\section{REFERÊNCIAS}


Anderson J. F. de Cerqueira, César V. de O. Carvalho Júnior, José M. Dias Filho

Ahrens, T., \& Mollona, M. (2007). Organisational control as cultural practice - A shop floor ethnography of a sheffield steel mill. Accounting, Organizations and Society, 32(4-5), 305-331. https://doi.org/10.1016/j.aos.2006.08.001

Apicella, C. L., Azevedo, E. M., Christakis, N. A., \& Fowler, J. H. (2014). Evolutionary origins of the endowment effect: $\quad$ Evidence from hunter-gatherers. American Economic Review, 104(6), 1793-1805. http://dx.doi.org/10.1257/aer.104.6.1793

Baskerville, R. F. (2003). Hofstede never studied culture. Accounting, Organizations and Society, 28(1), 1-14.

Bernartzi, S. \& Thaler, R. (1995). Myopic loss aversion and the equity premium puzzle. The Quarterly Journal of Economics, 110(1), 73-92. http://dx.doi.org/10.3386/w4369

Basu, S. (1997). The conservatism principle and the asymmetric timeliness of earnings. Journal of Accounting and Economics, 24, 3-37. https://doi.org/10.1016/S0165-4101(97)00014-1

Barberis, N. \& Hua N. G. (2001). Mental accounting, loss aversion, and individual stock returns, Journal of Finance, 56(4), 1247-1292. https://doi.org/10.3386/w8190

Birnberg, J. G., \& Snodgrass, C. (1988). Culture and control: A field study. Accounting, Organizations and Society, 13(5), 447-464. https://doi.org/10.1016/0361-3682(88)90016-5

Brasil - Geert Hofstede. ([s.d.]). https://geert-hofstede.com/brazil.html.

Busco, C., \& Scapens, R. W. (2011). Management accounting systems and organisational culture: Interpreting their linkages and processes of change. Qualitative Research in Accounting \& Management. 8(4), 320-357.

Carvalho, C., Albuquerque, F., Quirós, J., \& Justino, M. (2015). Uma análise das diferenças em termos da cultura a partir do projeto de substituição da IAS 39: Financial instruments - recognition and measurement. Revista de Educação e Pesquisa em Contabilidade, 9(1), 5-24. https://doi.org/10.17524/repec.v9i1.1112

Castro Neto, J. (1998). Contribuição ao estudo da prática harmonizada da contabilidade na União Europeia. 1998. Tese de Doutorado, Universidade de São Paulo, São Paulo, SP, Brasil.

Castro Neto, J., Itoz, C., Lima, I., Pasqual, D., Bastos, E. \& Muller, E. (2006). O Papel das culturas nacionais nas práticas contábeis do Brasil, Estados Unidos, França, Alemanha e Japão. Anais do Encontro da Anpad, Salvador, BRA, 30 .

Chand, P., Cummings, L. and Patel, C. (2012), The effect of accounting education and

national culture on accounting judgments: A comparative study of Anglo-celtic and Chinese culture. European Accounting Review, 21(1), 153-182. https://doi.org/10.1080/09638180.2011.591524

Chernick, M. R. \& Labudde, R. A. (2011). An introduction to bootstrap methods with applications to R. New Jersey: John Wiley e Sons.

Chow, C. \& Hwang, R. (1995) The Interaction between National Culture and Organisational Culture in Accounting Firms: Some Evidence from the Pacific Rim. Asia-Pacific Journal of Accounting, 2(1), 85-96. https://doi.org/10.1080/10293574.1995.10510479

Dickhaut, J. (2009). The brain as the original accounting institution. The Accounting Review, 84(6), 1703-171. http://dx.doi.org/10.2308/accr.2009.84.6.1703

Dickhaut, J., Basu, S., Mccabe, K. \& Waymire, G. (2010). Neuroaccounting: Consilience between the biologically evolved brain and culturally evolved accounting principles. Accounting Horizons, 24(2), 221-255. http://dx.doi.org/10.2308/acch.2010.24.2.221

Doupnik, T, S. \& Richter, M. (2004). The impact of culture on the interpretation of "in context" verbal probability expressions. Journal of International Accounting Research, 3(1), 1-20. https://doi.org/10.2308/jiar.2004.3.1.1

Doupnik, T. S. \& Riccio, E. L. (2006). The influence of conservatism and secrecy on the interpretation of verbal probability expressions in the Anglo and Latin cultural areas, The International Journal of Accounting, 41(3), 237-261. https://doi.org/10.1016/j.intacc.2006.07.005

Ellili, N. O. D. (2012). Managerial entrenchment: Model and impact on the shareholders' wealth. Corporate Ownership \& Control, 9(2-4), 449-460. http://dx.doi.org/10.22495/cocv9i2c4art6

Etemadi, H., Dilami, Z. D., Bazaz, M. S., \& Parameswaran, R. (2009). Culture, management accounting and managerial performance: Focus Iran. Advances in Accounting, 25(2), 216-225. https://doi.org/10.1016/j.adiac.2009.08.005 
Gray, S. (1988). Towards a Theory of cultural influence on the development of accounting systems internationally. Abacus, 1-15. https://doi.org/10.1111/j.1467-6281.1988.tb00200.x

Hair, J., Anderson, R., Tatham, R., \& Black, W. (2005). Análise multivariada de dados, (5. ed). New Jersey: Pearson.

Hall, M. (2008). The effect of comprehensive performance measurement systems on role clarity, psychological empowerment and managerial performance. Accounting, Organizations and Society, 33(2-3), 141-163. https://doi.org/10.1016/j.aos.2007.02.004

Han, S., Kang, T., Salter, S. \& Yoo, Y. K. (2010). A cross-country study on the effects of national culture on earnings management. Journal of International Business Studies, 41(1), 123-141. https://doi.org/10.1057/jibs.2008.78

Khlif, H. (2016). Hofstede's cultural dimensions in accounting research: A review, Meditari Accountancy Research, Emerald Group Publishing, 24(4), 545-573, October. http://dx.doi.org/10.1108/MEDAR-022016-0041

Hofstede, G., Bram, N. Ohayv, D. \& Sanders, G. (1990). Measuring organizational cultures: A qualitative and quantitative study across twenty cases. Administrative Science Quartely, 35(2), 286-316. http://dx.doi.org/10.2307/2393392

Hofstede, G. (1991). Cultures and organizations: Software of the mind. McGraw Hill: London.

. (2001). Culture's consequences, comparing values, behaviors, institutions, and organizations across nations. Thousand Oaks CA: Sage Publications.

Hofstede, G., Hilal, A., Malvezzi, S., Tanure, B. \& Vinken, H. (2010). Comparing regional cultures within a country: Lessons from Brazil. Journal of Cross-Cultural Psychology, 41(3), 336-352. https://doi.org/10.1177\%2F0022022109359696

Joannidés, V., Wichramasinghe, D., \& Berland, N. (2012), Critiques on Gray-Hofstede's model: What impact in cross-cultural accounting research?, In Conference Proceedings Comptabilités et innovations, Grenoble, France. http://hal.archivesouvertes.fr/hal-00690933/

Kahneman, D., Knetsch, J. L. \& Thaler, R. H. (1991). Anomalies: The endowment effect, loss aversion, and status quo bias. Journal of Economic Perspectives, 5(1), 193-206. https://doi.org/10.1257/jep.5.1.193

Kahneman, D. \& Tversky, A. (1979). Prospect theory: An analysis of decision under risk. Econométrica, 47(2), 263-291.

. Choices, values, and frames. (1983). American Psychologist, 39(4), 341-350.

Kang, Tony, LEE, Lian Fen, NG, Jeffrey, \& TAY, Joanne (2004). The Impact of Culture on Accounting Choices: Can Cultural Conservatism Explain Accounting Conservatism?. Asia Pacific Interdisciplinary Research in Accounting Conference 4th APIRA 2004, July 2-3. Research Collection School Of Accountancy.

Le Breton, D. (2009). As paixões ordinárias: Antropologia das emoções. Rio de Janeiro: Vozes.

Li, Y., Y. J., Kenrick, D., Griskevicius, V., \& Neuberg, S. (2012). Economic decision biases and fundamental motivations: How mating and self-protection alter loss aversion. Journal of Personality and Social Psychology, 102(3), 550-561. https://psycnet.apa.org/doi/10.1037/a0025844

Libby, R., Bloomfield, R., \& Nelson, M. (2002). Experimental research in financial accounting. Accounting, Organizations and Society, 27(8), 775-810. https://doi.org/10.1016/S0361-3682(01)00011-3

Lima, B. (2016). O impacto das dimensões culturais sobre a prática contábil no Brasil: Um olhar a partir da percepção dos operadores da contabilidade. Revista de Educação e Pesquisa em Contabilidade, 10(4), 363379. http://dx.doi.org/10.17524/repec.v10i4.1398

Lima Filho, R. N. \& Bruni, A. L. (2013). Quanto mais faço, mais erro? Uma análise sobre a presença de vieses cognitivos em julgamentos sobre orçamento. Revista Base (Administração e Contabilidade) da UNISINOS, 10(3), 224-239. https://10.4013/base.2013.103.03

Macêdo, J., Lopes, J., Silva, L., Ribeiro Filho, J., Pederneiras, M., \& Feitosa, M. (2010). Convergência contábil na área pública: Uma análise das percepções dos auditores de TCES, contadores e gestores públicos. Revista de Contabilidade e Organizações, 4(8), 69-91. https://doi.org/10.11606/rco.v4i8.34759 
Machado, M. \& Nakao, S. (2014). Influência das diferenças culturais, econômicas e sociais na adoção das IFRS. Revista Universo Contábil, 10(1), 104-125. https://doi.org/10.4270/RUC.2014106

Melo, C. \& Silva, C. (2010). Finanças comportamentais: Um estudo da influência da faixa etária, gênero e ocupação na aversão à perda. Revista de Contabilidade e Organizações, 4(8), 3-23. https://doi.org/10.11606/rco.v4i8.34756

Miguel, F. K. (2015). Psicologia das emoções: Uma proposta integrativa para compreender a expressão emocional. Psico-USF, Bragança Paulista, 20(1), 153-162. http://dx.doi.org/10.1590/1413-82712015200114

Novemsky, N. \& Kahneman, D. (2005b). How do intentions affect loss aversion? Journal of Marketing Research, 42(2), 139-140. https://doi.org/10.1509/jmkr.42.2.139.62295

Patel, C., Harrison, G. L., \& McKinnon, J. L. (2002). Cultural influences on judgments of professional accountants in auditor-client conflict resolution. Journal of International Financial Management \& Accounting, 13(1), p. 1-31. doi:10.1111/1467-646X.00077

Pompian, M. M. (2006). Behavioral finance and wealth management: How to build optimal portfolios that account for investor biases. New Jersey: John Wiley \& Sons.

Rebequi, T. (2015). Diferenças culturais brasileiras e suas implicações na experiência do usuário em interfaces web. Dissertação de Mestrado, Universidade de São Paulo, São Paulo, SP, Brasil.

Rieger, M., Wang, M., \& Hens, T. (2014). Risk preferences around the world. Management Science, 61(3), 637648. http://dx.doi.org/10.1287/mnsc.2013.1869

Rouziès, D., Segalla, M., \& Besson, M. (1999). The impact of cultural dimensions on sales force compensation, Jouy en Josas.

Schein, E. (1997). Organizational culture and leadership. San Francisco: Jossey-Bass.

Schultz, J. J. \& Lopez, T. J. (2001). The impact of national influence on accounting estimates: Implications for international accounting standard-setters. The International Journal of Accounting, 36(3), 271-290. https://doi.org/10.1016/S0020-7063(01)00103-0

Soeters, J. \& Schreuder, H. (1988). The interaction between national and organizational culture in accounting firms. Accounting Organizations and Society, 13(1), 75-85.

Sokol-Hessner, P., Hsu, M., Curley, N., Delgado, M., Camerer, C., \& Phelps, E. (2009) Thinking like a trader selectively reduces individuals' loss aversion. Proceedings of the National Academy of Sciences, 106(13), 5035-5040. https://doi.org/10.1073/pnas.0806761106

Sprinkle, G. B., Williamson, M. G., \& Upton, D. R. (2008). The effort and risk-taking effects of budget-based contracts. Accounting, Organizations and Society, 33(4), 436-452. https://doi.org/10.1016/j.aos.2007.11.001

Thaler, R. (2001). Dando aos mercados uma dimensão humana. Dominando finanças. São Paulo: Makron.

Thaler, R. \& Sunstein, R. (2008). Nudge: Improving decisions about health, wealth, and happiness. Yale University Press, New Haven: CT.

Tsui, J. S. (2001). The implication of culture on relationship between budgetary participation, management accounting systems, and managerial performance: An analysis of Chinese and Western managers. International Journal of Accounting (36), 125-146. https://doi.org/10.1016/S0020-7063(01)00101-7

Tversky, A. \& Kahneman, D. (1991). Loss aversion in riskless choice: A reference-dependent model. Quarterly Journal of Economics, 106(4), 1039-1061. https://doi.org/10.2307/2937956 . (1992). Advances in prospect theory: Cumulative representation of uncertainty. Journal of Risk and Uncertainty, 5(4), 297-323. https://doi.org/10.1007/BF00122574

Wang. M., Rieger, M., \& Hens, T. (2016). The impact of culture on loss aversion. Journal of Behavioral Decision Making, 30(2), 270-281. 


\section{APÊNDICE}

\section{Questionário sobre atitudes de risco em ambiente de perda}

Convido-o(a) a participar voluntariamente desta pesquisa, cujo objetivo é "compreender a percepção de risco". Garanto o anonimato, sigilo, privacidade e confidencialidade das questões respondidas, sem o risco de que seus dados individuais sejam identificados. Em qualquer etapa da pesquisa você terá o direito de se retirar do estudo, sem qualquer penalidade ou prejuízo. Suas informações serão analisadas junto com as de outros participantes. Diante desses esclarecimentos, assinale uma das duas alternativas a seguir:

Li e declaro minha ciência sobre os procedimentos desta pesquisa e minha disposição de participar voluntariamente.

Não concordo em participar (Neste caso, encerre sua participação nesta pesquisa).

Informe o seu nome

Descrição da Pesquisa

O objetivo desta pesquisa busca compreender o comportamento dos indivíduos, em situação de risco e incerteza, para tomar decisões de acordo com a influência cultural. A ideia é você imaginar, na realidade, até que ponto é possível arriscar em uma aposta.

Nos seguintes jogos você tem $50 \%$ de chance de ganhar ou perder dinheiro. Por favor, indique a quantidade MÍNIMA de dinheiro, no jogo A e B, para qual você estaria disposto a ganhar para participar do jogo A e B.

JOGO A

$50 \%$ de chance

de perder $\$ 25$

$50 \%$ de chance de ganhar $\$ \mathbf{X}$

Qual o valor MÍNIMO de \$ X você estaria disposto a ganhar para participar do jogo A?

JOGO B

$50 \%$ de chance

de perder $\$ 100$

$50 \%$ de chance de ganhar $\$ \mathbf{Y}$

Qual o valor MÍNIMO de \$ Y você estaria disposto a ganhar para participar do jogo? 
Ao responder a esta parte do questionário, pense num emprego IDEAL e procure considerar os fatores que seriam importantes numa situação IDEAL de trabalho. Não considere a importância que têm no seu trabalho atual.

Marque uma resposta em cada linha

1 - De muito pouca ou nenhuma importância

2 - De pouca importância

3 - De importância moderada

4 - De muita importância

5 - Da máxima importância

Em relação a cada ponto, responda à pergunta geral: QUE IMPORTÂNCIA TEM PARA SI...

Ter tempo suficiente para a minha vida pessoal e familiar

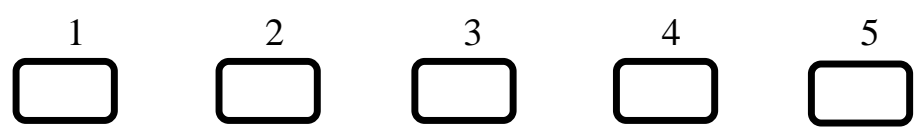

Ter boas condições físicas de trabalho (boa ventilação, luz, espaço adequado, etc.)

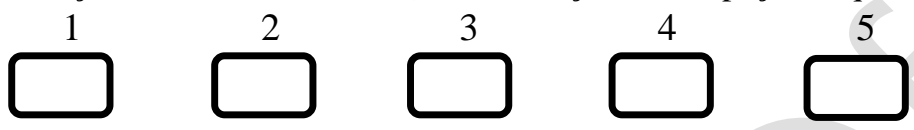

Ter boas relações com o seu superior direto

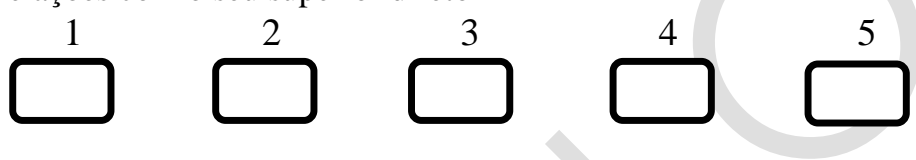

Ter segurança no emprego

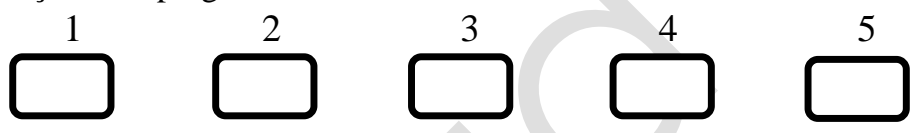

Trabalhar com pessoas que cooperam bem entre si

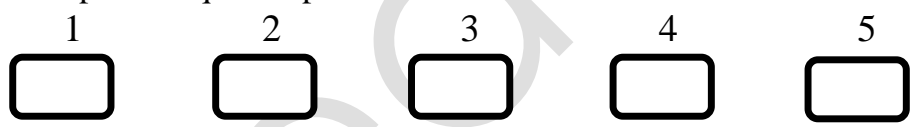

Ser consultado pelo seu (sua) superior direto (a) nas decisões que ele (ela) toma

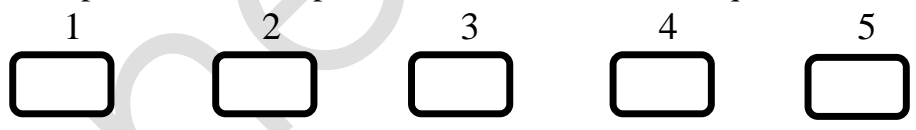

Ter oportunidade de avançar na carreira profissional para níveis mais elevados

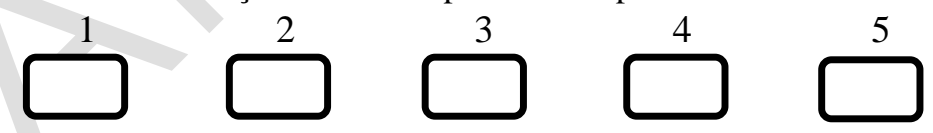

Ter elementos de variedade e aventura no trabalho

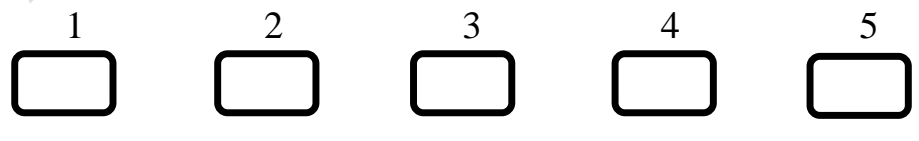




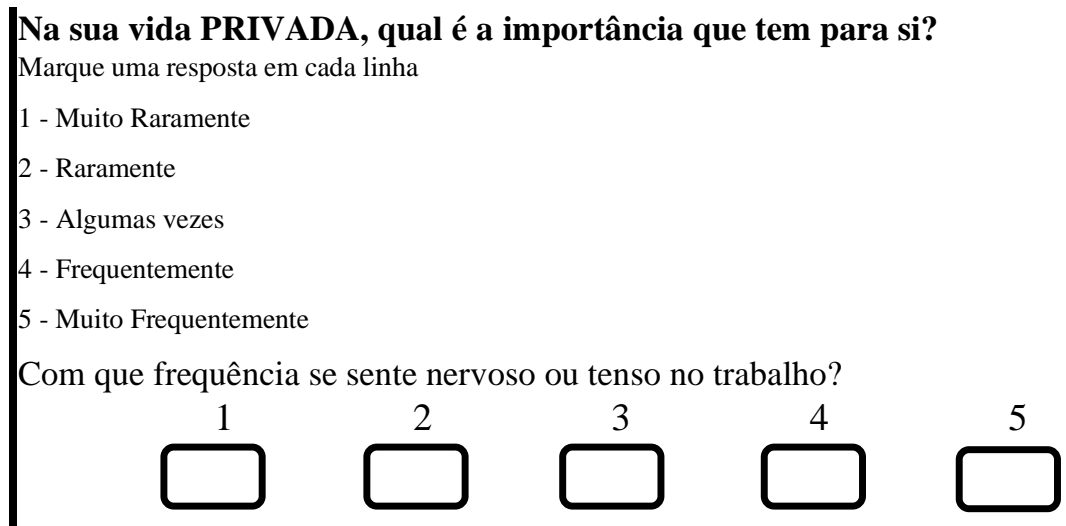

No seu ambiente de trabalho, com que frequência é que os subordinados receiam expressar em desacordo com os seus superiores?

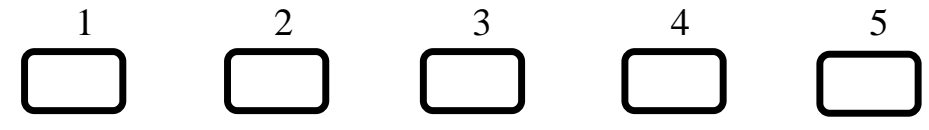

Por favor indique o seu grau de acordo ou desacordo relativo às seguintes afirmações:

Marque uma resposta em cada linha

1 - Discordo Inteiramente

2 - Discordo

3 - Indeciso

4 - Concordo

5 - Concordo Inteiramente

A maior parte das pessoas é de confiança

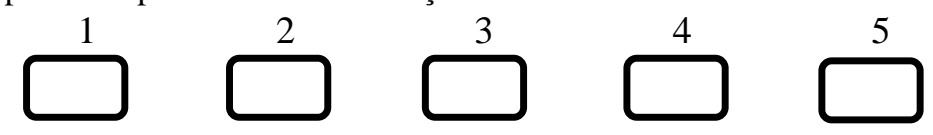

Pode-se ser um bom gestor sem ter respostas precisas à grande maioria das questões que os subordinados possam colocar à cerca do seu trabalho

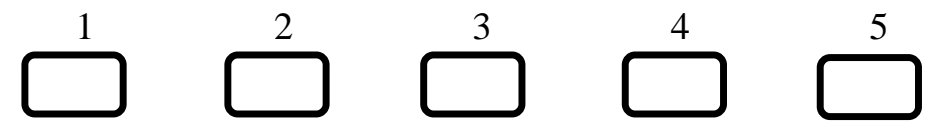

Uma estrutura organizacional certos subordinados têm duas chefias deve ser evitada a todo o custo

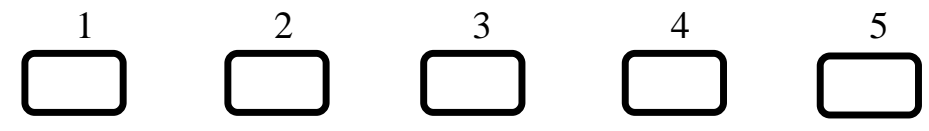

A competição entre empregados normalmente é mais negativa que positiva

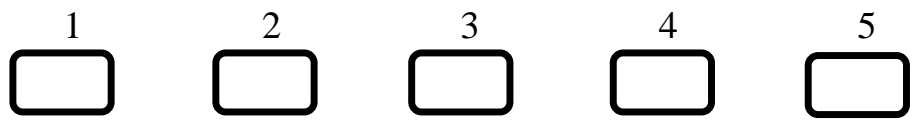

As normas de uma empresa não devem ser quebradas, mesmo que o empregado pense que é no melhor interesse da empresa

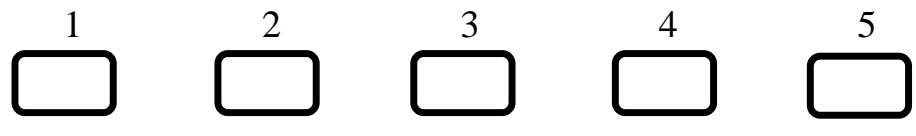


Anderson J. F. de Cerqueira, César V. de O. Carvalho Júnior, José M. Dias Filho

Quando as pessoas fracassam na sua vida, muitas vezes, é por sua culpa

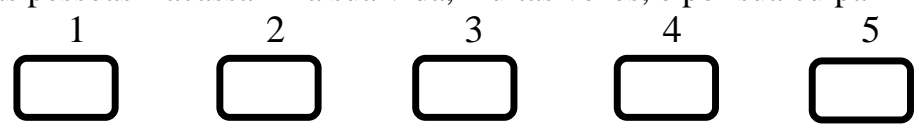

Sexo

Masculino

$\square$ Feminino

$\square$ Não desejo informar

Idade

Informe a sua atual área de desempenho profissional 